\title{
Effectiveness of a Brief Teaching Scenario in a Phantom-Based Learning Model for Students to Achieve Ultrasound-Guided Vascular Access-a Prospective Study
}

\author{
Philippe Jagoda $^{1}$ (D) Jonas Stroeder ${ }^{1} \cdot$ Arno Buecker $^{1} \cdot$ Joshua Gawlitza $^{1} \cdot$ Felix Frenzel $^{1} \cdot$ Paul Raczeck $^{1}$
}

Received: 8 September 2020 / Accepted: 22 October 2020 / Published online: 2 November 2020

(C) The Author(s) 2020, corrected publication 2021

\begin{abstract}
For students, early hands-on experience is very limited and often non-existent during study time. Thus, we aimed to evaluate the progress of inexperienced medical students in successfully establishing an ultrasound-guided vessel access. One brief, condensed single teaching lesson in a prior to post-teaching scenario was performed using an inexpensive, self-made phantom model. In this prospective study, medical students $(n=11)$ with no experience in ultrasound imaging performed an ultrasound-guided vessel access simulated by a gelatin-based phantom model. Success rates and time of procedures were measured. Afterwards, participants underwent dedicated supervised teaching in a single lesson (duration $30 \mathrm{~min}$ ) with both theoretical information given and practical training skills shown. Then, every student performed the very same procedure again and results were compared with paired $t$ test. Success rate of guide wire placement rose from 36.4 (4/11) to $100 \%$. Mean number of attempts significantly decreased with $2.5 \mathrm{SD} 1.3$ before and $1.2 \mathrm{SD} 0.4$ after teaching $(p<0.05)$. Overall time to successful guide wire placement improved from $291 \mathrm{SD} 8$ to $151 \mathrm{SD} 37 \mathrm{~s}(p<0.05)$. With already limited training time and opportunities available during medical education, short and simple, but highly effective training tools are invaluable. With the help of an inexpensive, self-made gelatinbased phantom model for ultrasound-guided vascular access, medical students demonstrate significantly improved practical puncture skills after only one brief, condensed teaching lesson and thus an important progress with regard to their future clinical routine. The performance of ultrasound-guided vascular access can be highly improved for inexperienced medical students by applying one short teaching session using an inexpensive, self-made phantom model.
\end{abstract}

Keywords Ultrasonography $\cdot$ Interventional $\cdot$ Phantoms $\cdot$ Imaging $\cdot$ Problem-based learning

Philippe Jagoda

philippe.jagoda@uks.eu

Jonas Stroeder

Jonas.Stroeder@uks.eu

Arno Buecker

Arno.Buecker@uks.eu

Joshua Gawlitza

Joshua.Gawlitza@uks.eu

Felix Frenzel

Felix.Frenzel@uks.eu

Paul Raczeck

Paul.Raczeck@uks.eu

1 Clinic for Diagnostic and Interventional Radiology, Saarland University Medical Center, Kirrberger Str. 1, 66421 Homburg/ Saar, Germany

\section{Introduction}

Ultrasound-guided needle placement for medical interventions is widely used by physicians throughout many medical specializations and is a very well established procedure [1-3]. Multiple clinical trials have shown a significant decrease in complications as well as a significantly improved success rate of needle punctures if performed with ultrasound guidance $[4$, 5]. Particularly, adequate needle tip visualization during ultrasound guidance plays the most important role in successfully performing the desired intervention compared with the traditional landmark technique without ultrasound [6]. Therefore, guidelines for ultrasound-guided vascular cannulation have been implemented in order to optimize vascular access procedures in patients [7].

However, for students, early hands-on experience is very limited and often non-existent during study time. The main reason for this is the lack of opportunities for realistic practice, 
because "trial and error" scenarios for warranted catheter placements in (critical care) patients are at least inadequate and often not acceptable to be performed by relatively inexperienced staff members, particularly students. Moreover, learning time is also limited and particularly during work as medical doctor, effectiveness of short but condensed training or teaching tools is warranted.

Therefore, the aim of this study was to evaluate the progress of inexperienced students to successfully perform ultrasound-guided vascular cannulation with only limited amount of teaching time and training accessible. Furthermore, we intended to use an inexpensive, relatively easy to manufacture phantom model.

\section{Material and Methods}

\section{Study Design}

This was a prospective trial of novice ultrasound users trying to achieve a real-time ultrasound-guided vascular access on a phantom. The participation of the medical students was on a voluntary basis. Every student was allowed to refuse to participate in the study without any disadvantages in the offered ultrasound course. Institutional review board approval was waived after consultation with the responsible ethics committee. Informed consent was not applicable for this in vitro study as no patients were included.

\section{Study Setting and Participants}

This trial was conducted at our institutional ultrasound training course. The ultrasound course is offered every semester and consists of 12 sessions with duration of 90 min, respectively. The number of participants is deliberately limited to 12 students in order to ensure a good teacher to student ratio for supervision. Two supervisors (senior physician in radiology, 8 and 11 years old experience) were responsible for the performance, instruction and observation of the procedures. The participants included 11 medical students (undergraduates; male/female ratio $=2 / 9$, mean age 22 years, range 20-26) with little to no experience with ultrasound usage and no experience in ultrasound-guided interventional procedures. In order to avoid gaining experience of the students in ultrasound, we decided to carry out this trial at the first two sessions of the ultrasound course.

Due to the lack of comparable literature data, no statistical power analysis with regard to the number of participants was possible.

\section{Study Protocol}

In a first session, all participants were asked to perform ultrasound-guided needle cannulation after being given brief oral instructions of how to use and hold the transducer and how the silicone tube is visualized in B-mode images (Mindray, M7 Diagnostic Ultrasound System, 9 MHz linear transducer). Subjects did not receive prior hands-on training and had very limited knowledge in theoretical aspects of ultrasound and ultrasound-guided interventional procedures before participation.

The students were briefed to handle puncture needles with extreme caution, and their handling was supervised at all times.

In the first session, the first task was to search, to identify and to visualize the silicone tube in the gelatin phantom and to depict the short and long axes - in that order (see Fig. 1) —of the tube in B-mode.

Then, an 18-gauge introducer steel needle was inserted under real-time ultrasound visualization (see Fig. 2). Duration time of both short and long axis depictions, time from first phantom tissue puncture to successful vessel

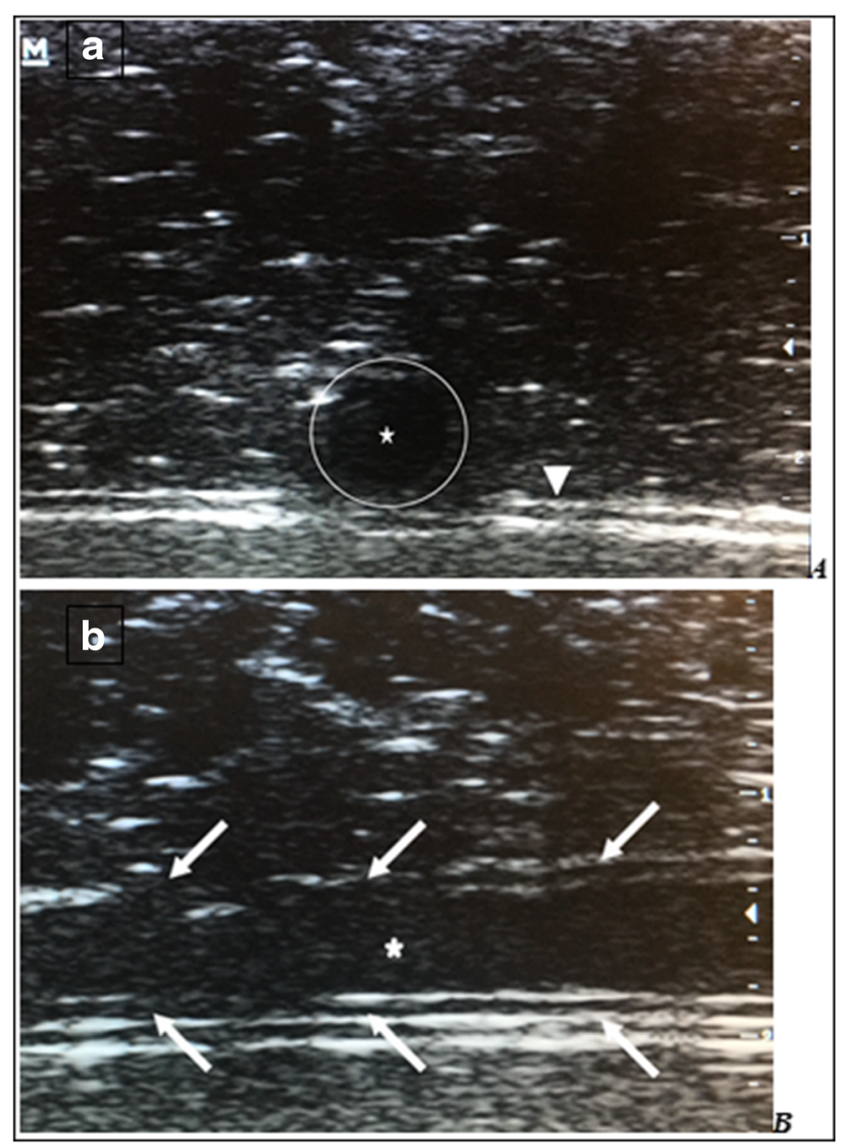

Fig. 1 Short (a) and long (b) axis visualization of the silicone tube in Bmode; circle, outer border of the tube in short axis; asterisk, inner lumen of the tube; triangle, bottom of the gelatin-based phantom model, arrows, outer border of the tube 


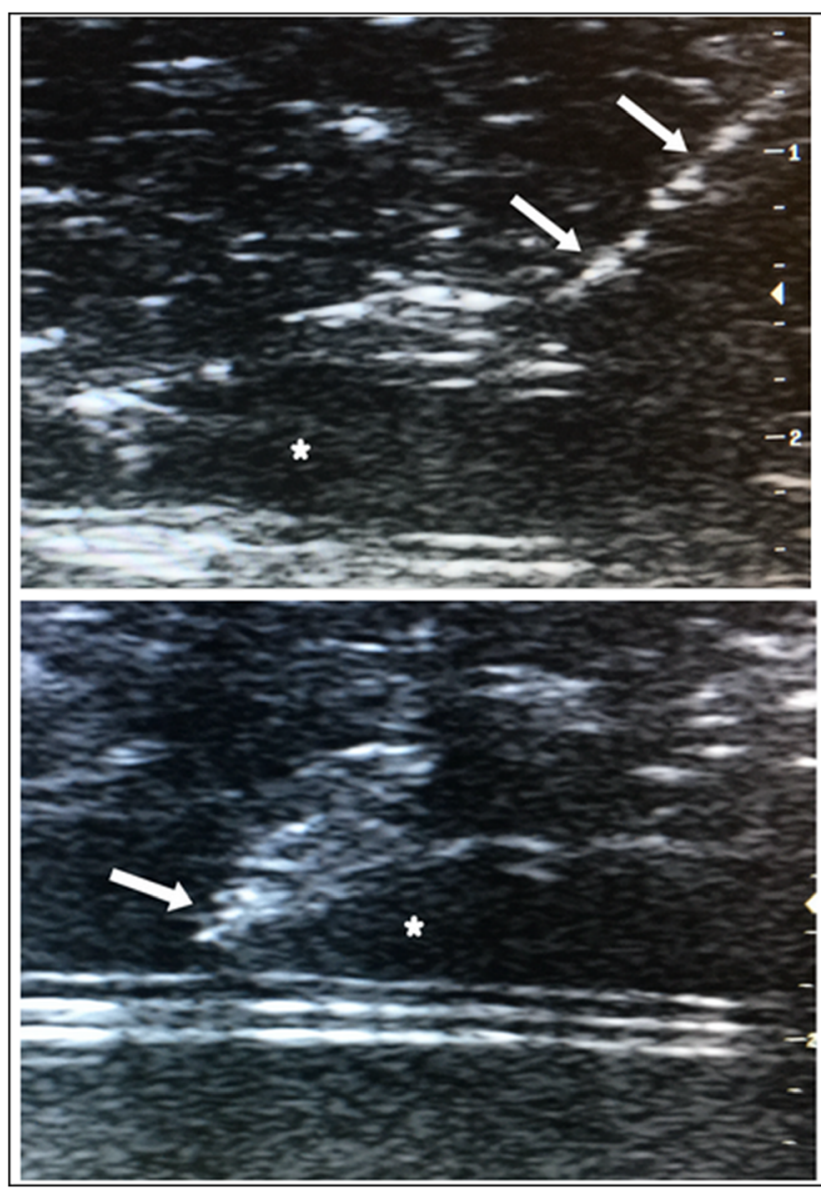

Fig. 2 Visualization of a steel needle cannulation in B-mode; arrows, steel needle; asterisk, inner lumen of the tube. In the upper picture, the needle tip is directly adjacent to the upper wall of the tube. In the lower picture after successful cannulation, the needle tip is visualized inside the lumen of the tube

puncture (as judged by a guide wire tip visualization at the end of the silicone tube sticking out of the gelatin container) as well as the number of attempts to puncture the silicone tubes were recorded. There was no time limit given, although all students who failed to insert guide wire placement aborted the procedure after about $10-15 \mathrm{~min}$ by themselves. Within this time interval, the students had any number of puncture attempts. If the cannulation was successful, the experiment was finished for the respective student. The duration of the first session was $2 \mathrm{~h}$.

Afterwards, all participants took part in a 30-min didactic session consisting of a dedicated PowerPoint presentation of ultrasound-guided procedures including physics, basic image adjustment and knowledge of medical instruments encompassing the ultrasound device as well as puncture needles. Additionally, example video clips and images of ultrasound-guided vascular access on the used tissue phantom as well as in real patients (such as central venous catheter placement, femoral artery punctures) were demonstrated.
During the didactic teaching lesson mentioned above, no hands-on training was offered.

In a second session, which equaled the first session in equipment, duration and set up, all students performed the procedure again and the aforementioned parameters were again recorded. No further assistance was given by supervisors in the second session.

Results of both practical sessions were compared in a prior to post-test scenario.

\section{Gelatin-Based Phantom Model}

The phantom model is related to comparable gelatin-based phantoms used and previously described by Clemmensen et al. $[8,9]$.

All materials necessary for creating the phantom model were available in regular stores, and supermarkets and overall costs were very low. The total sum added up to \$10-15.

Total ingredients list is as follows: water (1250 total volume), unflavoured gelatin powder ( $35 \mathrm{~g}$ per $250 \mathrm{~mL}$ volume; $0.30 €$ ), sugar-free Metamucil ( $15 \mathrm{~g}$ per $250 \mathrm{~mL}$ volume; 0.49 $€$ ), silicone tubes (length variable; $30 \mathrm{~cm}$, wall thickness 0.5 $\mathrm{mm}$, inner diameter $6 \mathrm{~mm} ; 1.50 €)$, aseptic alcohol-based solution $(\sim 1-2 €)$, blue colour additive $(0.69 €)$ and adhesive tape for fixation (Tesa; $1.50 €$ ). The silicone tubes were chosen to achieve the best haptic feedback; this was tested by subjective assessment of the authors.

The first step in preparing the phantom models was to create a gelatin mixture (Dr. Oetker gelatin powder) of 1.25 $\mathrm{L}$ volume. Thus, for every $250 \mathrm{~mL}$ volume, $35 \mathrm{~g}$ of gelatin powder was mixed with $250 \mathrm{~mL}$ of hot water just below boiling point. The gelatin was completely dissolved in water before one tablespoon (15 g) of Metamucil for every $250 \mathrm{~mL}$ of water was added. These fibres mimic the echo-density or scattering of human tissue seen with ultrasound. Also, $1 \mathrm{~mL}$ of colour additive (commercially available food colouring; blue) was added to assure invisibility of the silicone tubes below the gelatine surface.

When partially cooled down, $15 \mathrm{~mL}$ of alcohol-based antiseptic solution (chlorhexidine $0.5 \%$ in alcohol $70 \%$, Orphi Farma) per $250 \mathrm{~mL}$ of water was added to render antiseptic properties and to improve durability. An alcohol cleaned plastic container $(14 \mathrm{~cm} \times 24 \mathrm{~cm} \times 8 \mathrm{~cm})$ was used, and thin and smooth silicone tubes (wall thickness $0.5 \mathrm{~mm}$, inner diameter $6 \mathrm{~mm}$ ) were placed and fixed near the bottom of the container approximately $5 \mathrm{~cm}$ below the gelatin surface with the help of adhesive tape. Both ends of the silicone tube stick out of the plastic container to preserve visual control of guide wire placement, and the silicone tube was filled up with water to assure ultrasound visibility similar to a vessel. Finally, the gelatin solution described above was filled into the container and cooled down in a refrigerator $\left(5^{\circ} \mathrm{C}\right)$ for $3 \mathrm{~h}$ until the gelatin was stiffened. Figure 3 shows the phantom-based 


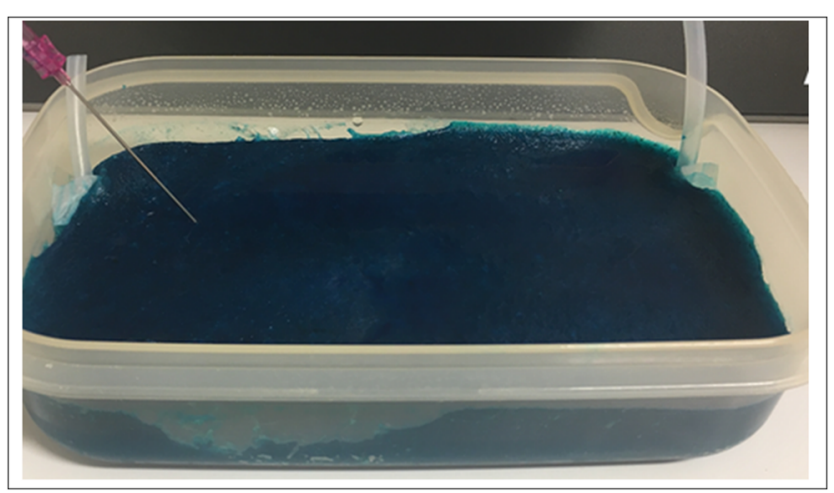

Fig. 3 The gelatin-based phantom model with an 18-gauge steel needle which was used for cannulation. Both ends of the silicone tube are depicted on the left and right and stick out of the gelatin mixture

gelatin model. Due to the preservation by the alcohol, the identical phantom could be used for all experiments.

\section{Statistical Analysis}

Time (in sec.) and number of attempts were documented for each participant before and after theoretical and practical training. Data was analysed concerning normal distribution by using a Kolmogorov-Smirnov test. Paired $t$ test was used to evaluate the difference in performance for normally distributed data; $p<0.05$ was applied for statistical significance.

\section{Results}

Before going into detail regarding statistics, we like to share some interesting subjective observations we made before any teaching and training had taken place: some students were unable to hold the transducer in an appropriate way, not rectangular to the phantom surface and were unable to combine visual and motor skill. In detail, they were unable to watch the screen of the ultrasound device and handle the transducer at the same time. Other students did not manage to identify the tube underneath the surface even though they correctly executed the visual and motor skills mentioned above.

Results of both training sessions before and after dedicated teaching lessons are depicted in Figs. 4 and 5, respectively.

Normal distribution was confirmed by using the Kolmogorov-Smirnov analysis with KS distances ranging between 0.1448 and 0.2270 , with each $p$ value not stating a statistically significance ( $p>0.1$ for all distributions).

In the first session without any proper training or teaching, only 4 out of 11 students (36.4\%) managed to somehow place the guide wire correctly into the silicone tube.

Mean number of attempts was $2.5+-1.3$, mean time to short axis visualization was $52+-36 \mathrm{~s}$, time to long axis visualization was $67+-61 \mathrm{~s}$, time to tube perforation was

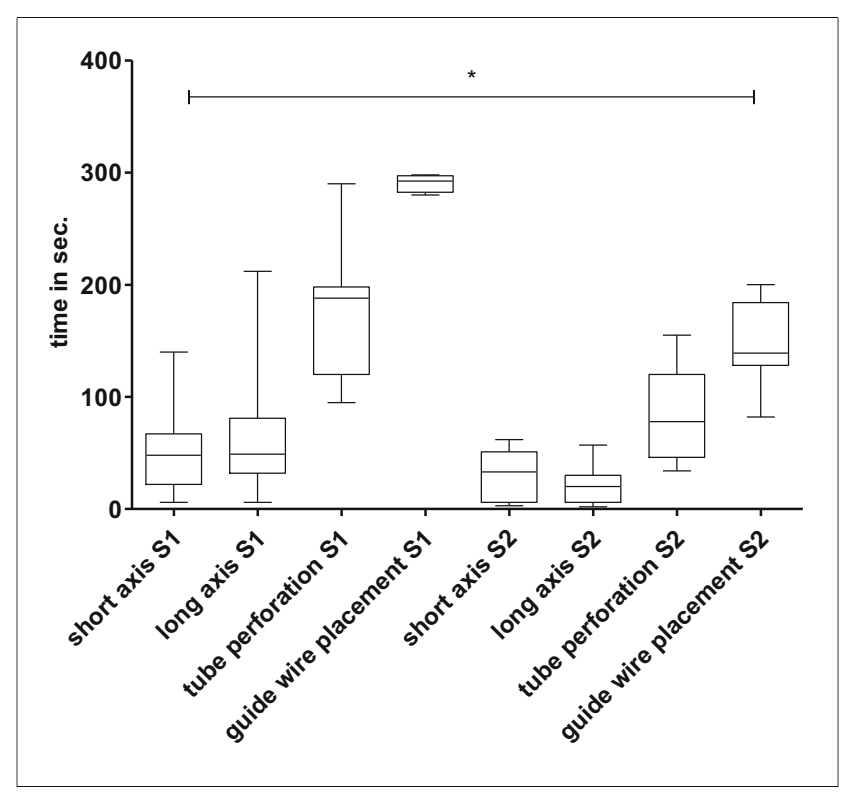

Fig. 4 Comparison of procedural durations: The vertical lines represent standard deviation with mean indicated as a horizontal line. The $25 \%$ and $75 \%$ quartiles are depicted as a box plot. *Statistically significant with $p<$ 0.05 for all comparisons between session 1 and session 2

$167+-59 \mathrm{~s}$ and time to successful guide wire placement (applicable only in 4 out of 11 participants) was $291+-8 \mathrm{~s}$.

In the second session after dedicated didactic lesson, success rate rose to $100 \% ; 11$ out of 11 students correctly placed the guide wire into the silicone tube.

Mean number of attempts were $1.2+-0.4(p=0.0222)$. Mean time using short axis visualization was reduced to $31+$ $-22 \mathrm{~s}(p=0.0414)$. Time to long axis visualization was reduced to $21+-17 \mathrm{~s}(p=0.0113)$, and time to tube perforation diminished to $82+-39 \mathrm{~s}(p=0.0003)$. Time to successful guide wire placement was significantly reduced to 151 $+-37 \mathrm{~s}(p=0.0038)$ with 11 out of 11 participants.

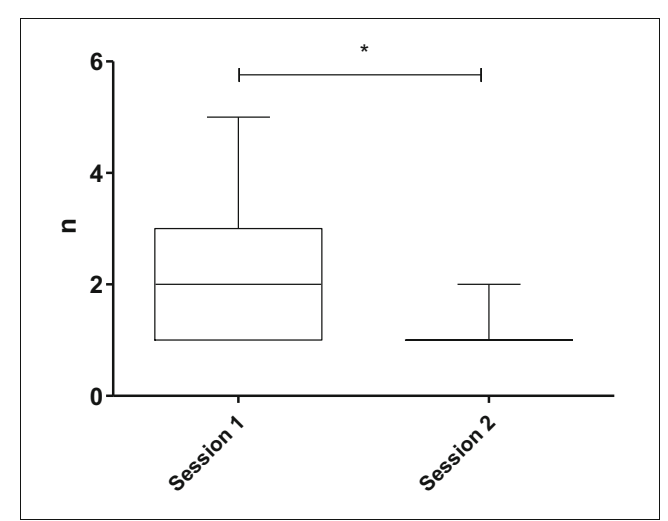

Fig. 5 Comparison of number of cannulation attempts: The vertical lines represent standard deviation with mean indicated as a horizontal line. The $25 \%$ and $75 \%$ quartiles are depicted as a box plot. *Statistically significant with $p<0.05$ 
In our results, statistically significant differences were found for every comparison measured between the first and the second hands-on training sessions.

\section{Discussion}

Our results indicate a highly beneficial impact of a single, brief but condensed didactic lecture and a supervised short hands-on training session in a simple phantom model with regard to successfully establishing an ultrasound-guided vascular access. Procedural times in ultrasound visualization and number of cannulation attempts showed a statistically significant decrease indicating a highly improved practical proficiency in our student group. Moreover, success rate of ultrasound-guided endoluminal guide wire placement rose from 36.4 to $100 \%$; thus, every student was able to perform the procedure.

These results are comparable with the review of literature by Gottlieb et al. [10], wherein between 89.1 and $97.7 \%$ of medical students $(n=198)$ of three independent studies successfully performed cannulation and consecutive guide - wire placement via short or long axis imaging.

Likewise, McKay and Weerasinghe demonstrated that teaching basic interventional ultrasound skills to novice junior doctors in a single focused session is an achievable outcome with very high success rates of up to $99 \%$ of the students [11].

In accordance to our results, in the study group of Vitto et al. [12], 100\% of participants using real-time ultrasound guidance successfully achieved cannulation. After theoretical and practical training, Vitto et al. also had nearly identical mean number of attempts $(n=1.31)$ compared with our study collective $(n=1.2)$.

Moreover, Wagner et al. [13] showed with thirty-nine inexperienced physicians significantly decreasing rates of failure of guide-wire insertion into 2-mm tubes after dedicated teaching courses, using both long axis $(p=0.001)$ and short axis imaging $(p=0.004)$ techniques. The numbers of successful cannulations on the first attempt increased after the teaching in all methods ( $p=0.001)$, a result to be considered negatively proportional to our statistically significantly reduced mean number of attempts.

In contrast to our trial, however, the teaching scenarios cited in above-mentioned publications comprised either a factory-produced, commercially available and thus relatively expensive phantom model or much higher amount of teaching time with often multiple sessions of hands-on training conducted. Our set-up, on the other hand, was able to show equally significant results with both financially and temporally very low efforts.

Multiple training scenarios have been described and deemed equally effective with regard to impact on novice's progress in ultrasound skills [14-16].
In general, training must include image acquisition, image interpretation, real-time use of ultrasound and an experienced instructor in order to develop motor skills with the gain of a haptic feedback. Additionally, education should include a combination of didactic lectures with live videos or photos of real vascular access cannulation guided by ultrasound [17].

This trial had some limitations. Due to the lack of strictly comparable literature data, no statistical power analysis was possible. Nevertheless, the relatively low number of participants yielded already highly significant results and thus the necessity of additional participants obsolete. Also, since hands-on practice played an important role for this study, a larger group size could also have led to a decrease in practical proficiency in this trial setup due to limited amount of ultrasound devices and phantom models available. Due to ethical constrains, the highly interesting effect in clinical practice on patients could not be tested in our scenario.

The gelatin phantom model differs from human tissue and the silicone tube used could not perfectly mimic the human vasculature. Therefore, haptic feedback was slightly different while using artificial silicone tubes compared with human veins or arteries. Moreover, image quality of gelatin-based tissues is much lower compared with "in vivo" tissue. Stone et al. [6] could show ultrasound images with relatively high image quality in factory-produced, tissue models, although its costs will most likely be much higher than ours.

Regardless of these differences, the beneficial impact of phantom models has been previously described by Clemmesen et al. $[8,9]$ and likewise our results strongly indicate skill improvement, which was the primary aim of this study.

Moreover, further differentiation between the effect of hands-on practice on the phantom model itself and implementing theoretical knowledge of concept could not be stated. It is likely that practice on the phantom would be the key driver for improved success when assessing an interventional performance, but better theoretical knowledge of how to approach the procedure would also improve performances as well. Furthermore, it is debatable that prior exposure to the phantom in the first session would independently act to improve the results of the second session even without theoretical knowledge at all.

But it is important to mention that neither a statistical comparison between theoretical learning effect and practical skill development nor distinguishing between both aforementioned effects was subject of our evaluation.

As a conclusion, a phantom-based training model for ultrasound-guided vascular procedures using real-time ultrasound is highly recommended as a dedicated training tool. The production of our gel phantom is relatively easy, fast and inexpensive. Moreover, a single, brief but condensed theoretical teaching session combined with the aforementioned phantom model suffices for success of novice trainees. 
The truly low cost for the phantom and the short time of teaching capacity needed should allow to introduce our examined set up into medical training for all students and should be made available wherever and whenever possible to improve vascular cannulation skills.

\section{Lessons for Practice}

- Novice ultrasound users greatly benefit from one condensed, brief teaching scenario in an inexpensive, selfmade phantom model to successfully establish an ultrasound-guided vessel access.

- Suitable, inexpensive phantom models for hands-on training in ultrasound-guided vessel punctures can be individually designed and easily manufactured under trivial conditions.

- With minimal teaching effort and low cost invested, a highly beneficial impact on interventional skill improvement can be shown in this teaching scenario.

Acknowledgements The authors would like to thank all medical students who participated in this study and gave consent for both participation and publication of their learning results.

Authors' Contributions P.J. initiated the conception of the study, participated in the primary acquisition of medical students and helped in the analysis and interpretation of data for this study. P.R. helped in the acquisition of medical students, performed the analysis and first interpretation of data for this study and performed the initial drafting the manuscript and first critical revision of the manuscript. P.J. and P.R. created the phantom model used for the student's interventions. J.G., F.F. and J.S. participated in the critical review of the manuscript and acquisition of data. A.B. participated in the critical revision of the manuscript. All authors gave final approval of the submitted manuscript and agreed on its publication.

Funding Open Access funding enabled and organized by Projekt DEAL.

Data Availability All data generated or analysed during this study are included in this published article.

The materials used can be found in the "Material and Methods" section under the "Gelatin-Based Phantom Model" subsection (total ingredients list).

\section{Compliance with Ethical Standards}

Ethics Approval and Consent to Participate We have sent our study project to the responsible ethics committee. According to e-mail correspondence, our study does not require a written ethics committee approval according to the "Ärztekammer des Saarlandes" (responsible ethics committee). We have added our e-mail correspondence with the ethics committee as a separate PDF file. No ethics approval applicable, since novice ultrasound users applied real-time ultrasound-guided vascular access on a gelatin phantom model. Neither institutional review board approval nor informed consent was necessary for this in vitro study as no patients were included. All medical students involved in this study gave consent to participate and to publish their learning results.
Consent for Publication All medical students involved in this study gave consent for publication.

Competing Interests The authors declare that they have no competing interests.

Open Access This article is licensed under a Creative Commons Attribution 4.0 International License, which permits use, sharing, adaptation, distribution and reproduction in any medium or format, as long as you give appropriate credit to the original author(s) and the source, provide a link to the Creative Commons licence, and indicate if changes were made. The images or other third party material in this article are included in the article's Creative Commons licence, unless indicated otherwise in a credit line to the material. If material is not included in the article's Creative Commons licence and your intended use is not permitted by statutory regulation or exceeds the permitted use, you will need to obtain permission directly from the copyright holder. To view a copy of this licence, visit http://creativecommons.org/licenses/by/4.0/.

\section{References}

1. Miller AH, Roth BA, Mills TJ, Woody JR, Longmoor CE, Foster B (2002) Ultrasound guidance versus the landmark technique for the placement of central venous catheters in the emergency department. Acad Emerg Med 9:800-805. https://doi.org/10.1111/j.1553-2712. 2002.tb02168.x

2. Milling TJ Jr, Rose J, Briggs WM, Birkhahn R, Gaeta TJ, Bove JJ, Melniker LA (2005) Randomized, controlled clinical trial of pointof-care limited ultrasonography assistance of central venous cannulation: the Third Sonography Outcomes Assessment Program (SOAP-3) Trial. Crit Care Med 33:1764-1769. https://doi.org/10. 1097/01.ccm.0000171533.92856.e5

3. Karakitsos D, Labropoulos N, De GE, Patrianakos AP, Kouraklis G, Poularas J, Samonis G, Tsoutsos DA, Konstadoulakis MM, Karabinis A (2006) Real-time ultrasound-guided catheterisation of the internal jugular vein: a prospective comparison with the landmark technique in critical care patients. Crit Care 10:R162. https:// doi.org/10.1186/cc5101

4. Hilty WM, Hudson PA, Levitt MA, Hall JB (1997) Real-time ultrasound-guided femoral vein catheterization during cardiopulmonary resuscitation. Ann Emerg Med 29:331-336. https://doi.org/10. 1016/s0196-0644(97)70344-5

5. Lamperti M, Bodenham AR, Pittiruti M, Blaivas M, Augoustides JG, Elbarbary M, Pirotte T, Karakitsos D, Ledonne J, Doniger S, Scoppettuolo G, Feller-Kopman D, Schummer W, Biffi R, Desruennes E, Melniker LA, Verghese ST (2012) International evidence-based recommendations on ultrasound-guided vascular access. Intensive Care Med 38:1105-1117. https://doi.org/10. 1007/s00134-012-2597-x

6. Stone MB, Moon C, Sutijono D, Blaivas M (2010) Needle tip visualization during ultrasound-guided vascular access: short-axis vs long-axis approach. Am J Emerg Med 28:343-347. https://doi. org/10.1016/j.ajem.2008.11.022

7. Troianos CA, Hartman GS, Glas KE, Skubas NJ, Eberhardt RT, Walker JD, Reeves ST (2011) Guidelines for performing ultrasound guided vascular cannulation: recommendations of the American Society of Echocardiography and the Society of Cardiovascular Anesthesiologists. J Am Soc Echocardiogr 24: 1291-1318. https://doi.org/10.1016/j.echo.2011.09.021

8. Clemmesen L, Knudsen L, Sloth E, Bendtsen T (2012) Dynamic needle tip positioning - ultrasound guidance for peripheral vascular access. A randomized, controlled and blinded study in phantoms 
performed by ultrasound novices. Ultraschall Med 33:E321-E325. https://doi.org/10.1055/s-0032-1312824

9. Clemmesen L, Bendtsen TF, Sloth E, Oveland NP, Knudsen L (2013) Gelatine phantom for training of ultrasound guided vascular access. Ugeskr Laeger 175:576-578 https://europepmc.org/article/ $\operatorname{med} / 23608008$

10. Gottlieb M, Holladay D, Peksa GD (2018) Comparison of short- vs long-axis technique for ultrasound-guided peripheral line placement: a systematic review and meta-analysis. Cureus 10:e2718. https://doi.org/10.7759/cureus. 2718

11. McKay GFM, Weerasinghe A (2018) Can we successfully teach novice junior doctors basic interventional ultrasound in a single focused training session? Postgrad Med J 94:259-262. https://doi. org/10.1136/postgradmedj-2018-135590

12. Vitto MJ, Myers M, Vitto CM, Evans DP (2016) Perceived difficulty and success rate of standard versus ultrasound-guided peripheral intravenous cannulation in a novice study group: a randomized crossover trial. J Ultrasound Med 35:895-898. https://doi.org/10. 7863/ultra.15.06057

13. Wagner M, Hauser K, Cardona F, Schmölzer GM, Berger A, Olischar M, Werther T (2018) Implementation and evaluation of training for ultrasound-guided vascular access to small vessels using a low-cost cadaver model. Pediatr Crit Care Med 19:e611e617. https://doi.org/10.1097/PCC.0000000000001721

14. Feller-Kopman D (2007) Ultrasound-guided internal jugular access: a proposed standardized approach and implications for training and practice. Chest 132:302-309. https://doi.org/10.1378/chest. 06-2711

15. Skippen P, Kissoon N (2007) Ultrasound guidance for central vascular access in the pediatric emergency department. Pediatr Emerg Care 23:203-207. https://doi.org/10.1097/PEC. 0b013e3180467780

16. Chenkin J, Lee S, Huynh T, Bandiera G (2008) Procedures can be learned on the Web: a randomized study of ultrasound-guided vascular access training. Acad Emerg Med 15:949-954. https://doi. org/10.1111/j.1553-2712.2008.00231.x

17. Moro C, Smith J, Stromberga Z (2019) Multimodal learning in health sciences and medicine: merging technologies to enhance student learning and communication. Adv Exp Med Biol 1205: 71-78. https://doi.org/10.1007/978-3-030-31904-5_5

Publisher's Note Springer Nature remains neutral with regard to jurisdictional claims in published maps and institutional affiliations. 